\title{
The adipose-derived Nerve Growth Factor is associated with abdominal obesity in prepubertal and pubertal children
}

\author{
Pilar Argente Arizón ${ }^{1,2}$, Azahara Iris Rupérez ${ }^{1,2}$, Concepción Aguilera $^{3,4}$, Rosaura Leis ${ }^{5,4}$, \\ Mercedes Gil Campos ${ }^{6,4}$, Ángel Gil ${ }^{3,4}$, Luis Alberto Moreno ${ }^{1,4}$ and Gloria Bueno ${ }^{7,4}$ \\ ${ }^{1}$ Growth, Exercise, NUtrition and Development (GENUD) Research Group, University of Zaragoza, Zaragoza, Spain, \\ ${ }^{2}$ Health Research Institute of Aragón (ISS Aragón), Agroalimentary Institute of Aragón (IA2), Zaragoza, Spain, \\ ${ }^{3}$ Department of Biochemistry and Molecular Biology II, Instituto de Nutrición y Tecnología de los Alimentos, \\ Universidad de Granada; Instituto de Investigación Biosanitaria ibs, Granada, Spain, \\ ${ }^{4}$ Spanish Biomedical Research Centre in Physiopathology of Obesity and Nutrition (CIBERobn), Madrid, Spain, \\ ${ }^{5}$ Galicia Nutrition, Growth and Human Development Research Unit, Pediatric Department; Instituto de Investigación \\ Sanitaria de Santiago de Compostela (IDIS); Santiago de Compostela, Spain, \\ ${ }^{6}$ Paediatric Research and Metabolism Unit, Hospital Universitario Reina Sofia; Instituto Maimónides de Investigación \\ Biomédica (IMBIC), Córdoba, Spain and \\ ${ }^{7}$ Paediatric Department, Lozano Blesa University Hospital, University of Zaragoza, Zaragoza, Spain
}

\begin{abstract}
Introduction: Obesity is known to be associated with a low-grade inflammatory state. Most studies reporting inflammation in obesity have been done in pubertal children or adults or have focused only on the relation between body mass index (BMI) and the classical inflammatory markers. Nerve growth factor (NGF), monocyte chemoattractant protein-1 (MCP-1) and hepatocyte growth factor (HGF) are adipokines previously shown to be involved in the inflammatory context of obesity and metabolic syndrome but few studies have been conducted in children.
\end{abstract}

Objective: To evaluate the association between NGF, MCP-1, HGF with total and abdominal adiposity and in prepubertal and pubertal children.

Methods: 889 children participated in a prospective obesity case-control study $(50.2 \%$ males, $72 \%$ prepubertals, $27 \%$ overweight and $43 \%$ obesity), paired by age and sex. The relationship between total fat (assessed using the BMI, the sum of skinfolds thickness (SF) and fat mass index (FMI) from bioelectrical impedance analysis and waist circumference as a surrogate marker of abdominal obesity) and plasma concentrations of MCP-1, HGF, and NGF was studied in prepubertal and pubertal children.

Linear regression analysis for prepubertal and pubertal children, adjusting for age, sex and center was used.

Results: NGF was associated with WC, with a stronger association in prepubertal than in pubertal children. MCP-1 was positively associated with WC, SF, FMI and BMI in pubertal children whereas HGF was positively associated with WC and BMI only in prepubertal children. The strongest associations were between WC and NGF $(\beta 0.12395 \% \mathrm{CI} 0.133,0.709 ; \mathrm{p}<0.001)$ in prepubertal children and WC and MCP-1 ( $30.12395 \%$ CI $0.438,2.035 ; \mathrm{p}<0.001)$ in pubertal children.

Discussion: Total and abdominal adiposity are related to plasma concentrations of adipokines in prepubertal and pubertal children with obesity. Adipokines such as NGF, HGF and MCP-1 have not been so well studied so far in obese children. Particularly, NGF was associated with WC and BMI in prepubertal children and with WC in pubertal children. The association between this neurotrophin, secreted by adipose tissue and involved in the development and survival of sympathetic neurons, with adiposity, especially in prepubertal children, could suggest an anti-inflammatory mechanism and thus be a potential therapeutic target.

\section{Conflict of interest}

None

\section{Conflict of Interest}

There is no conflict of interest 